\title{
Whatever happened to the axion?
}

\author{
The continuing absence of the particle called the axion is an embarrassment for particle physicists, \\ especially because it is improbable that its discovery need wait on new accelerators.
}

EVER since Dirac's daring suggestion, in 1929 , that the symmetry of what seemed to him a properly relativistic wave equation for the electron made the existence of its positively charged partner, the posiron, essential, particle physicists have grown used to the notion that new particles are more often predicted than discovered unexpectedly. Thus it was with Yukawa's mediator of the strong nuclear interaction (eventually identified as the pion), the anti-proton, the omega $\Omega^{ \pm}$ meson, the charmed quark (otherwise the $J / \psi$ ) and, most recently and spectacularly, the heavy bosons that mediate the weak nuclear interaction known as $Z^{0}$ and $W^{ \pm}$. The few surprises have been the recognition that the 'mesotrons' of cosmic-ray physics in the late $1930 \mathrm{~s}$ included not merely the expected pions but the heavy analogues of electrons now called muons, the discovery of strange particles in the late 1940s and the later but less surprising discovery of the third generation of leptons, the tauon.

None of this is surprising. Finding a novel particle is not child's play, but almost always a matter of searching for a meagre signal against a rich background of distracting particle transformations. Rarely are the predictions of the existence of a new particle accompanied by estimates of the mass as accurate as those for the bosons of the weak interaction, with the consequence that people must go hunting through the energy spectrum before they find a particle that generally fits the bill. Only then can the properties of the particle be defined so as to tell the circumstances in which they are likely to be observed.

Unfortunately, this line of argument does not provide a suitable explanation for the continued absence of the particle called the axion, an electrically neutral object whose mass is probably the equivalent of at most a few electron-volts and which should be recognizable by its decay into two photons which, because the total energy available is limited, would be recognizable in the ultraviolet. On the face of things, detection should be a simple matter, not requiring the availability of great particle energy from a frontline accelerator. But the axion remains elusive.

That such a particle should exist stems from the observation, originally by Lee and Yang more than a quarter of a century ago, that parity (evenness or oddness of a state) is not invariably conserved in transformations effected by the weak nuclear interaction. This entirely unexpected happening, mirrored a little later by the recognition that, in the decay of the 'strange' meson $K^{\circ}$, the principle seems not to apply that the laws of physics are invariant when the direction (or algebraic sign) of time is reversed, seems however to be exclusive to the weak interaction. The strong nuclear interaction, by contrast, faithfully respects both parity and time reversal.

Particle physicists are entirely familiar with such states of affairs. If there is one rule for one set of circumstances and another for a second, those such as particle physicists whose goal is to find a unified description of phenomena speak of there being an underlying symmetry which has somehow been broken in the exceptional case. If, as seems sensible, they require that the results of calculations from the laws of physics should be independent of the arbitrary complex numbers the elementary equations may embody, which is what the notion of gauge symmetry entails, each broken symmetry requires the existence of a particle, or perhaps a family of particles. The axion emerges from such a chain of argument. Technically, it must be a boson, with integral, not half-integral spin, and it must have some mass, which may however be very small.

As will by now be familiar, axions are potentially convenient particles. Indeed, over the past few years, they have been widely canvassed as candidates for the WIMPs (weakly interacting massive particles) whose unremarkable presence in the Universe in very large numbers might provide the missing mass required to hold the Universe together. From time to time, there have been reports of ultraviolet background radiation from distant regions of the Universe suggestive of axion decay, but none has carried conviction. Curiously, it has fallen to astrophysicists rather than to cosmologists and particle physicists to pin down the properties of axions a little more precisely. Because of the weak interactions involved in thermonuclear fusion, stars should be prolific sources of axions, whence upper limits can be placed on their mass (unlikely to exceed $25 \mathrm{eV}$, or 0.001 per cent of the mass of an electron). But axions must certainly interact weakly with other matter, while the half-life of their spon- taneous decay into a pair of photons must be long, probably exceeding the present lifetime of the Universe.

So what hope can there be of finding evidence of axions? Thomas W. Kephart and Thoman J. Weiler of the Vanderbilt University at Nashville, Tennessee, had an ingenious idea (Phys. Rev. Lett. 58, $171 ; 1987)$ almost exactly a year ago. They suggested that because axions have mass, they should congregate together in clusters. Even though their lifetime is expected to be very long, there is no reason why their combined radiation of decay photons should not be perceptible, to say the least of it.

Indeed, Kephart and Weiler worked out in their formal calculation that, in spite of the very long lifetime of these objects, the sheer weight of numbers of axions in a cluster should give them a luminosity comparable with that of ordinary stellar matter. In short, people should have set out to look for diffuse patches of luminosity in the sky whose total light output might be comparable with that of the Sun, but which would seem much less intense because of their physical extent. (Because axions interact only weakly with other matter and only gravitationally with themselves, they are not easily cooled and thus condensed.) What the calculations showed is that axion light should be detectable from the Milky Way or from external galaxies such as Andromeda provided that axions account for one part in a million of the observable mass, which is modest by the cosmologists' expectations.

So what has happened in the intervening year? Nothing much, it seems. The great astronomy journals have not been filled with reports of axion searches, successful or otherwise. Nor, apparently, have people been much concerned to elaborate the calculations so as to suggest where in the sky searchers should most efficiently point their telescopes. One difficulty, that should eventually be a benefit, is that the radiation from an axion cluster might simply appear as a very narrow and very faint ultraviolet line that would escape detection so long as the mass is so ill-defined. But there is also a chance that the axion does not exist (there are other candidates for the role of the allpervading WIMP), in which case there will be a scandal in particle physics. Should not that danger be taken more seriously?

John Maddox 